\title{
Self-organized colloidal quantum dots and metal nanoparticles for plasmon-enhanced intermediate-band solar cells
}

\author{
Manuel J Mendes, Estela Hernández, Esther López, \\ Pablo García-Linares, Iñigo Ramiro, Irene Artacho, Elisa Antolín, \\ Ignacio Tobías, Antonio Martí and Antonio Luque
}

\begin{abstract}
A colloidal deposition technique is presented to construct long-range ordered hybrid arrays of self-assembled quantum dots and metal nanoparticles. Quantum dots are promising for novel opto-electronic devices but, in most cases, their optical transitions of interest lack sufficient light absorption to provide a significant impact in their implementation. A potential solution is to couple the dots with localized plasmons in metal nanoparticles. The extreme confinement of light in the near-field produced by the nanoparticles can potentially boost the absorption in the quantum dots by up to two orders of magnitude.

In this work, light extinction measurements are employed to probe the plasmon resonance of spherical gold nanoparticles in lead sulfide colloidal quantum dots and amorphous silicon thin-films. Mie theory computations are used to analyze the experimental results and determine the absorption enhancement that can be generated by the highly intense near-field produced in the vicinity of the gold nanoparticles at their surface plasmon resonance.

The results presented here are of interest for the development of plasmon-enhanced colloidal nanostructured photovoltaic materials, such as colloidal quantum dot intermediate-band solar cells.
\end{abstract}

(Some figures may appear in colour only in the online journal)

\section{Introduction}

During the last decade, research on semiconductor nanoparticles, also known as quantum dots (QDs), has been growing enormously, mostly stimulated by their potential for opto-electronic applications such as solar cells [1-4], photodetectors [5-7], semiconductor lasers [8], light-emitting devices, etc. QDs are attractive candidates for the building blocks of nanostructured functional materials, since their nanometric dimensions allow the size-tuning of their electronic band structure due to quantum-confinement effects.
The optical properties of interest for most of the previously mentioned applications are those related to electronic transitions (by photon absorption or emission) to or from the QD conduction band edge, i.e. involving the QD lowest energy confined states above its bandgap. However, usually the measured absorption of the dots associated with such transitions is quite small, one or more orders of magnitude lower than that of their bulk material $[5,9-11]$. So, to better exploit the characteristics of QDs, it is important to couple them with light-trapping structures optimized to amplify the light intensity in their volume, at the energies of 


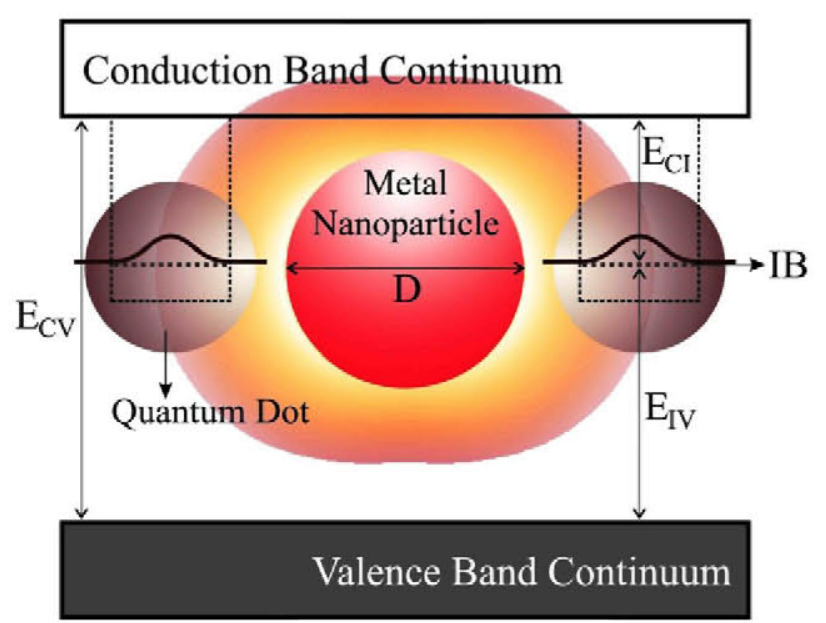

Figure 1. Schematic band diagram of a semiconductor material with embedded colloidal quantum dots (CQDs) immersed in the near-field produced by a metal nanoparticle (MNP). The MNP acts as a dipolar antenna, gathering the incoming light from its surroundings and focusing it in its localized near-field. The set of confined ground-states (dotted lines) of the QDs can form what is known as an intermediate band (IB). The IB allows the production of photocurrent from below-bandgap-energy photons since it enables electrons to be pumped from the valence band to the IB (across $E_{\mathrm{IV}}$ ) and from the IB to the conduction band (across $E_{\mathrm{CI}}$ ). For the fabrication of actual devices, the MNPs should be coated with a thin insulating shell, in order to prevent electron-hole recombination at the metal surface.

the optical transitions of interest, in order to compensate for their poor absorption $[4,12-18]$.

The work presented here investigates a way of realizing this goal using the strong electrostatic near-field produced in the vicinity of metal nanoparticles (MNPs) assembled side-by-side with QDs. Each MNP acts as an effective dipolar antenna at its localized plasmon resonance, bringing the incident energy from its surroundings and focusing it in its intense near-field where the QDs are located (sketched in figure 1) [7, 8, 19-21].

The interaction between excitons and plasmons, in hybrid structures of semiconductor and metal nanoparticles, has attracted significant attention in the last few years due to the variety of interesting effects that have been observed [7, 22], such as energy transfer, enhanced luminescence, etc. In particular, several contributions showed enhanced photoluminescence $[8,20,21,23,24]$, due to plasmon-enhanced absorption at or above the QDs ground-state, when the exciting light matches the plasmonic resonance of appropriately designed MNPs located in a near-field vicinity of the dots. The interaction with the MNPs usually results, however, in a decrease of the luminescence lifetimes of the QDs [24]. Despite such lifetime reduction, silver nano-islands formed by thin-film annealing have been shown [5] to increase the near-infrared photocurrent produced by QD films in photodetectors. These findings demonstrate the potentialities of near-field plasmon-enhanced QD absorption to improve the light harvesting properties of nanostructured opto-electronic devices.
In this paper, a novel metal-semiconductor nanoparticle system is studied composed of self-assembled arrays of colloidal MNPs and colloidal QDs (CQDs), patterned with controllable inter-particle distances, that can extend throughout indefinitely large sample areas. Such structure can be deposited on the surface of distinct materials through fast and inexpensive processes (by dip or spin coating, inkjet printing, among others).

Optical measurements were performed to study the light extinction enhancement caused in lead sulfide $(\mathrm{PbS})$ CQDs and in amorphous silicon (a-Si) thin-films by an array of single gold $(\mathrm{Au}) \mathrm{MNPs}$ sustaining surface plasmons (SPs). The MNPs are synthesized in colloidal solution with approximately spherical shapes [25], so their electric-field pattern is analytically modeled using Mie theory [26-28]. This is an electromagnetic separation-of-variables method that can compute scattering by particles of any size, but is limited to spherical geometries. The computational results provide important insight into the nature of the measured light extinction caused by the MNPs, and determine the maximum amount of absorption enhancement that they can produce in the material located in their near-field proximity.

The results presented in this paper can contribute to the development of colloidal-nanostructured photovoltaic materials. One particularly promising application is in quantum dot intermediate-band solar cells (QD-IBSCs). As represented in figure 1, when QDs are embedded in a semiconductor with a higher bandgap, their confined ground-state level can allow the promotion of electrons from the valence to the conduction band of the host semiconductor through the absorption of two consecutive photons with energies below the host bandgap $\left(E_{\mathrm{CV}}\right)$. Therefore, QD-IBSCs are able to generate photocurrent from a higher portion of the solar spectrum relative to conventional single-gap cells, without voltage reduction $[2,29,30]$. QD-IBSCs have usually been fabricated with QDs epitaxially grown on III-V materials $[8,10]$. However, the use of colloidal QDs can be more advantageous, since they can be made of a broad range of materials, fabricated with the appropriate nanoscopic dimensions $[9,11]$, and assembled together with MNPs, as described in section 2, which can potentially enhance the light absorption in the dots by more than one order of magnitude at their plasmon resonance $[12,19]$. The CQDs of interest for application in IBSCs should be composed of a low-bandgap semiconductor material, such as the PbS CQDs $[1,3-5,7,31]$ used in this work.

\section{Hybrid arrays of quantum dots and metal nanoparticles}

Colloidal MNPs are usually stabilized in solution by organic capping agents attached to their surface which provide a negative surface charge to the particles, causing them to repeal each other and preventing their aggregation [25]. A wet-coating method was developed to self-assemble such colloids in long-range ordered arrays, on the surface of various materials (see section 6.1). That is achieved by chemically functionalizing the surface with molecular linkers 

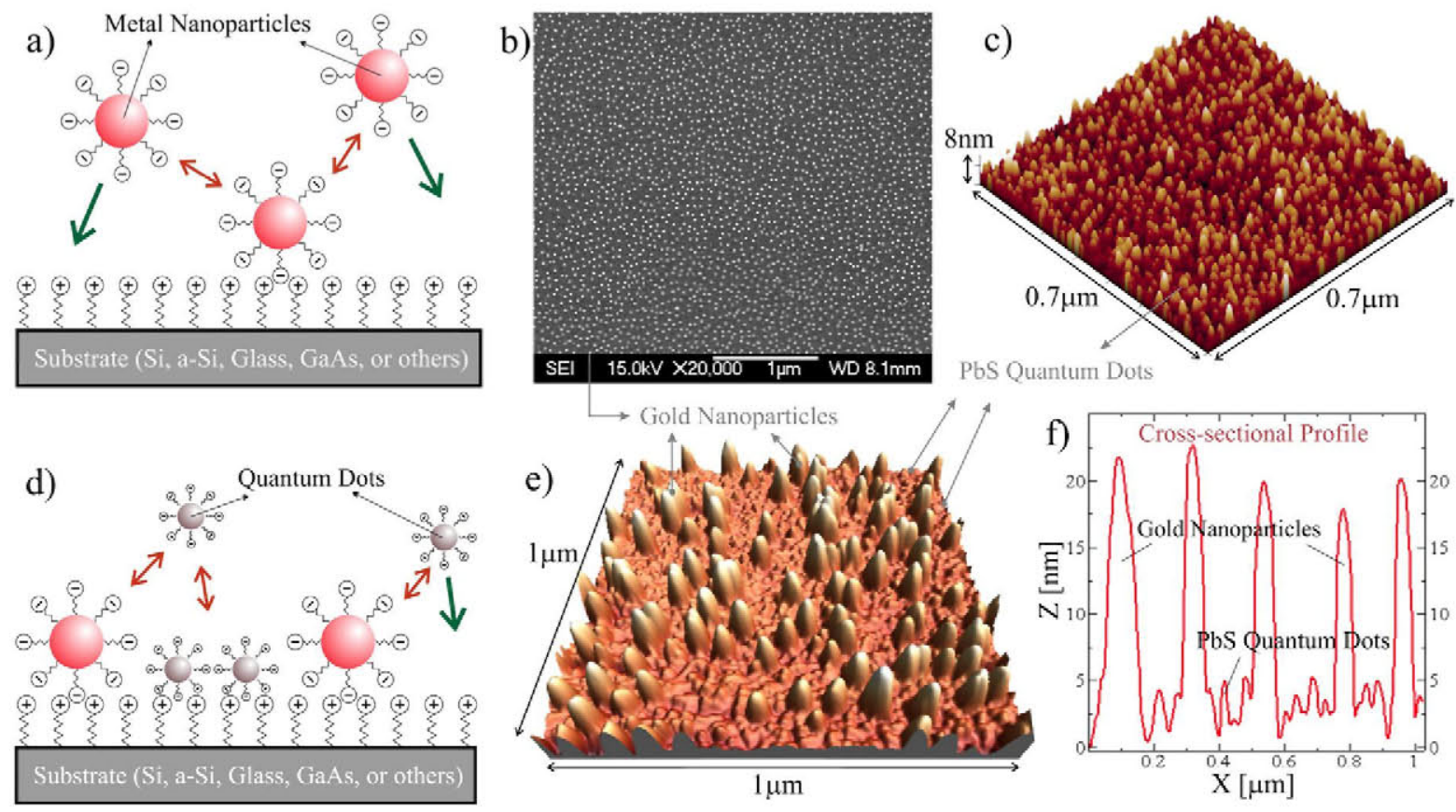

Figure 2. (a) Illustration of the self-organization of colloidal MNPs upon deposition. The MNPs are stabilized by negatively-charged capping agents, so they are attracted (green arrows) to any positively-charged surface. The electrostatic repulsion forces (red arrows) between the colloids establish a uniform inter-particle distance upon deposition. (b) Scanning electron microscope (SEM) image of an array of Au MNPs patterned on Si. The MNPs have a mean diameter of $20 \mathrm{~nm}$. (c) Atomic force microscope (AFM) image of a PbS CQD array on Si. The CQDs have a mean diameter of $5.1 \mathrm{~nm}$. (d) Sketch of the assembly of CQDs in the spaces between the previously deposited MNPs. (e) AFM image of an array of MNPs and CQDs patterned with this method (detailed in section 6.1). (f) Cross-section profile along a line of (e), where the heights of the MNPs and CQDs can be distinguished.

that have positively-charged end groups which attract the negatively-charged nanoparticles (see figure 2(a)) $[32,33]$. During the wet-coating process the MNPs are free to diffuse across the surface, so they settle in their lowest energy configuration which corresponds to a planar array as that shown in figure 2(b). It was verified that such structure can extend throughout the entire area of arbitrarily large samples, keeping a roughly uniform center-to-center (CtC) distance between the MNPs. This distance is proportional to the MNPs' surface charge density; so it can be tuned, for instance, by changing the capping agent, solvent liquid or $\mathrm{pH}$ of the solution.

The same procedure can be applied to the patterning of CQDs [6], allowing the construction of PbS CQD arrays as that shown in figure 2(c).

Upon sunlight illumination, an MNP scatters as a dipole [26] (illustrated in figure 1). Therefore, the region of highest electric-field intensity outside the particle occurs close to the MNP equator, in the direction of the incident electric-field vector. Such scattered near-field is evanescent, therefore it is only significant up to a distance of about the MNP radius outside the particle. So, the CQDs should be patterned as close as possible and in between the MNPs in order to take most profit from their localized short-ranged near-field.

The previously described deposition method allows the construction of such arrangement with controllable inter-particle separation distances. When a CQD solution is spin- or dip-coated onto a surface previously patterned with MNPs, such as that in figure 2(b), the CQDs deposit in the positively-charged surface areas between the MNPs separated by a certain distance established by the inter-particle electrostatic repulsion forces, as depicted in figure 2(d). Figure 2(e) shows an example of an hybrid array of MNPs and CQDs fabricated in this way, where the CQDs are deposited in the free areas between the MNPs. Vertical cross-sectional profiles, as that in figure 2(f), reveal that no CQDs are deposited on top of the MNPs: all the CQDs are assembled in the MNP inter-spaces as desired.

This wet-coating method is adaptable to practically any surface material, since most surfaces can be functionalized with positively-charged linkers. Therefore, arrays similar to those in figure 2 were fabricated, with particles of distinct sizes, on the surface of different materials that were tested: crystalline-Si wafers, a-Si films, gallium arsenide (GaAs) wafers and glass slides. The resulting nanoparticle arrays were also revealed to be independent of the surface doping, since similar distributions were imaged on intrinsic, n-type and p-type $\mathrm{Si}$ and GaAs wafers.

The possibility of integrating hybrid arrays of MNPs and CQDs in a broad range of host materials is an important advantage for their application in intermediate-band devices, since it facilitates the construction of plasmonenhanced CQD-IBSCs with the desired energy bandgaps ( $E_{\mathrm{IV}}, E_{\mathrm{CI}}, E_{\mathrm{CV}}$-represented in figure 1) required for the optimal performance of these cells [29]. 
An important aspect regarding the incorporation of colloidal particles in solar cells is that the organic substances (e.g. capping agents and linkers), involved in the colloids' fabrication and patterning processes, should be removed before embedding the particles in the cell material. These substances can deteriorate the electric response of the devices by decreasing the mobility and acting as trapping centers for the photo-generated carriers. However, due to their small molecular weight, such chemical residuals are volatile and may be removed under thermal annealing after depositing the colloids. According to tests performed by the authors, heat treatment can be performed at $200-300^{\circ} \mathrm{C}$ to remove the residuals present in our deposition process without affecting the patterned particles $[32,34]$.

Metallic surfaces are another main source of current degradation in solar cells, since metals act as recombination centers causing severe electron-hole recombination. Therefore, the surface of the MNPs should be coated with an insulating shell $[25,35]$ having a thickness on the order of the nanometer. This was not considered in the present studies, but would be important in the construction of actual solar cell devices $[36,37]$. Such shell reduces the intensity of the MNPs' scattered near-field, but can still allow them to produce significant absorption enhancement in their surrounding medium [19].

\section{Light extinction at plasmon resonance}

An MNP acts as an antenna for the incoming light, so it can collect the light from an area, known as the extinction cross section $\left(C_{\text {ext }}\right)$, higher than its physical area $\left(A_{\mathrm{MNP}}\right) . C_{\mathrm{ext}}$ is maximum at the surface plasmon (SP) resonance of the MNP, so the SP frequency can be determined by measuring the extinction (inverse of transmission) spectrum of an MNP array using spectrophotometry $[34,38]$ (see section 6.4).

Spectrophotometry is currently the most straightforward optical method to accurately determine the spectral position of the plasmon resonance of samples containing MNPs. Therefore, this technique is convenient for the optimization of experimental procedures involved in the fabrication of novel devices containing MNP structures. In this section, we analyze the results obtained by spectrophotometry of samples containing MNP arrays on glass and in CQDs and a-Si films. As explained below, such measurements do not allow the direct quantification of the light absorption enhancement produced by the MNPs in their surrounding material. However, they can still provide the main information, relative to the MNPs and their interaction with their dielectric environment, necessary for the optimization of the MNPs' relevant physical parameters.

\subsection{Colloidal metal nanoparticle arrays on glass}

Figure 3 shows the measured light extinction of MNP arrays, with particles of distinct diameter $(D)$, patterned onto glass slides with the method described in section 2. The MNP arrays give a reddish tone to the glass slides similar to the color of the MNP solutions.

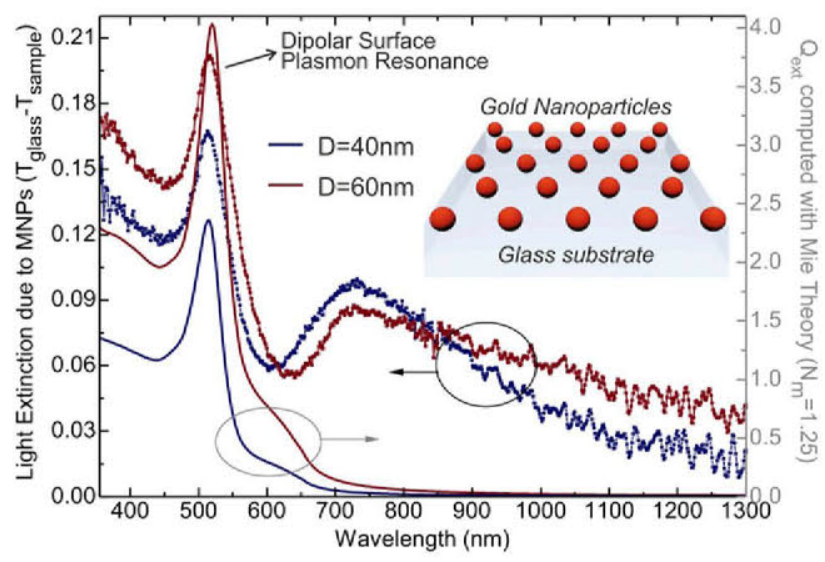

Figure 3. Measured extinction (left axis) of Au MNP arrays on glass, with particles of distinct diameter $(D)$. The plotted curves correspond to the extinction of MNPs-coated glass $\left(1-T_{\text {sample }}\right)$ minus that of just glass $\left(1-T_{\text {glass }}\right)$. For comparison, it is shown the extinction efficiency $Q_{\text {ext }}$ (right axis) of single MNPs inside a homogeneous medium with refractive index $N_{\mathrm{m}}=1.25$, calculated with Mie theory. The wavelengths $(\lambda)$ of the computed extinction peaks at the surface plasmon resonance $(\lambda=514 \mathrm{~nm}$ for $D=40 \mathrm{~nm}$ and $\lambda=518 \mathrm{~nm}$ for $D=60 \mathrm{~nm}$ ) match those measured experimentally.

The extinction spectra can be analytically modeled using Mie theory [26-28, 34], by calculating the extinction efficiency $\left(Q_{\text {ext }}=C_{\text {ext }} / A_{\mathrm{MNP}}\right)$ of a single MNP. Mie theory is only applicable to spherical particles assumed to be immersed in an infinite medium with a uniform refractive index $N_{\mathrm{m}}=n_{\mathrm{m}}+\mathrm{i} k_{\mathrm{m}}$. Nevertheless, in the samples of figure 3 the MNPs are deposited on the surface of a glass substrate in air. So, the medium that is surrounding the deposited MNPs can be modeled by an effective refractive index with a value between that of air $\left(N_{\mathrm{m}}=1\right)$ and glass $\left(N_{\mathrm{m}}=1.5\right)$. As such, the $Q_{\text {ext }}$ curves in figure 3 were computed considering $N_{\mathrm{m}}=1.25$, which yields extinction maxima precisely matched with the SP peaks of the optical measurements. However, in the measured curves there is an additional secondary peak at $\sim 730 \mathrm{~nm}$. This is a dispersive lineshape associated with the interaction between the MNPs and the substrate surface [39, 40]. Such peak is not present in the $Q_{\text {ext }}$ spectra because the effect of the refractive index discontinuity at the glass-air interface is not taken into account with Mie theory. Such discontinuity generates back-reflected fields from the glass surface which are not in phase with the MNPs' dipolar field, thus originating retarded potentials that cause the dispersive lineshape at wavelengths $(\lambda)$ above the SP resonance.

The $\mathrm{CtC}$ distance between the MNPs is of about three times their diameter, as determined from SEM images such as figure 2(b). Therefore, no inter-particle interaction effects are expected to be observed in the extinction measurements, since such effects only become significant for $\mathrm{CtC}$ distances lower than about twice the MNPs diameter [41].

\subsection{Plasmon-enhanced absorption in colloidal quantum dots}

The amount of light absorbed by a single monolayer of $\mathrm{PbS}$ CQDs, as that in figure $2(\mathrm{c})$, is so small that cannot be detected 


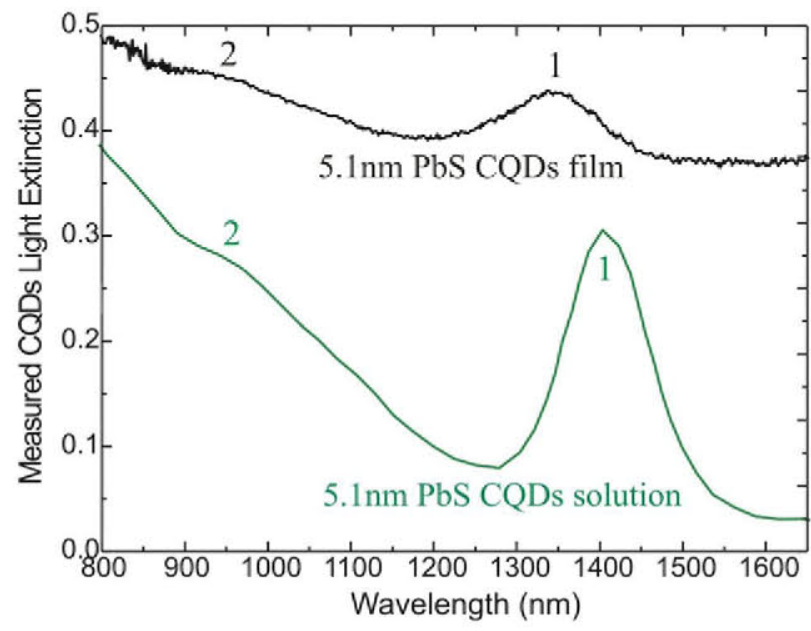

Figure 4. Measured extinction spectra of quantum dots while dispersed in colloidal solution (green line, in au) and after being deposited in a $\sim 1 \mu \mathrm{m}$ thick film supported on glass (black line). The absorption peak corresponding to the CQDs ground-state (labeled 1) is observed at $\lambda=1.4 \mu \mathrm{m}$ for the CQDs solution and at $\lambda=1.35 \mu \mathrm{m}$ for the film. The second excited state (labeled 2) can also be identified.

by standard optical measurements such as spectrophotometry. Therefore, to optically measure the light absorbed by the CQDs alone and compare it with that absorbed by the CQDs in the presence of MNPs, it is necessary to use several stacked CQD monolayers forming a CQDs thin-film (see section 6.2).

The absorption coefficient of PbS CQDs $[5,18]$ in the visible range is $\sim 10^{5} \mathrm{~cm}^{-1}$, and near their band edge is $\sim 10^{4} \mathrm{~cm}^{-1}$. However, the absorption coefficient of CQD films is usually lower; since the CQDs are spaced in the films by organic capping molecules (in our case, oleic acid) which absorb weakly at optical frequencies. So, film thicknesses of $\sim 1 \mu \mathrm{m}$ are required to clearly observe the extinction peak corresponding to the CQDs ground-state absorption [1]. This is shown in figure 4 together with the corresponding peak of the CQDs in solution.

Thin-films of $\mathrm{PbS}$ dots were deposited onto the MNP arrays of figure 3 to measure the light extinction that $\mathrm{Au}$ MNPs can produce in CQDs at the plasmon resonance. For the purposes of this work, we are interested in analyzing extinction effects due to single-particle scattering. Therefore, the thickness of these films is $\sim 100 \mathrm{~nm}$, which should be thin enough to prevent the establishment of photonic waveguided modes in the film [5] but still sufficiently thick to cover the MNPs' near-field region and to allow a measurable absorption in the visible range at the MNPs' SP wavelengths.

The results are given in figure 5, which shows the light extinction of samples supported on glass substrates that contain only the CQDs film and the film deposited over the MNPs arrays. As performed for the modeling of the SP peaks of figure 3 , the wavelengths of the extinction peaks match that of the $Q_{\text {ext }}$ peak, computed with Mie theory (see inset), considering an effective refractive index for the medium of $N_{\mathrm{m}}=1.72$. The value obtained for the effective $N_{\mathrm{m}}$ surrounding the MNPs should roughly correspond to the average between the refractive index of glass $\left(N_{\mathrm{m}}\right.$ (glass) $=$

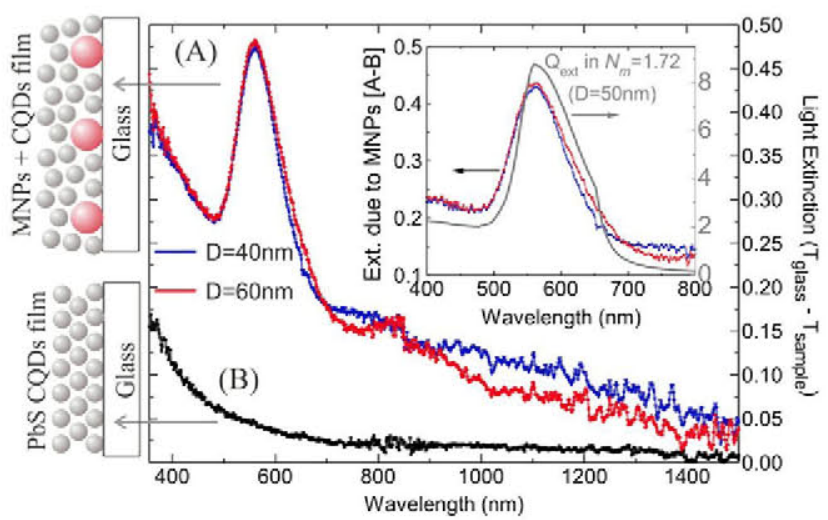

Figure 5. Extinction spectra of Au MNPs on glass after being covered by a PbS CQDs film with a thickness of about $100 \mathrm{~nm}$ (A). The black line (B) shows the spectrum of just the CQDs film deposited on glass. All the plotted curves correspond to the extinction of the samples $\left(1-T_{\text {sample }}\right)$ minus that of just glass $\left(1-T_{\text {glass }}\right)$. The inset shows the extinction of the MNPs + CQDs samples minus that of the CQDs film, which yields the amount of light that is extinct due to the presence of the MNPs. The measured extinction peaks at $\lambda=561 \mathrm{~nm}$ correspond to the MNPs plasmonic resonance, and match the $Q_{\text {ext }}$ peak (gray line) computed with Mie theory considering an effective refractive index of the medium $N_{\mathrm{m}}=1.72$.

1.5) and the real part of the refractive index of the CQDs film $\left(n_{\mathrm{m}}(\mathrm{CQDs})\right)$. So, this provides a first-order estimate for the value of $n_{\mathrm{m}}$ (CQDs) $=2.3$ at the SP wavelength, which is within the range of values reported in the literature [18]. The refractive index of the CQDs film also has an imaginary part $k_{\mathrm{m}}$ (CQDs), on the order of 0.01-0.1, which is responsible for the extinction (essentially caused by light absorption in the film) depicted in the black curve of figure 5. However, the imaginary part of the medium refractive index does not significantly shift the SP peak position, so it is not taken into account in the calculation of $Q_{\text {ext }}$ since it uniquely leads to an attenuation of the $Q_{\text {ext }}$ magnitude [18].

It is interesting to observe that the presence of the MNPs in the CQDs film leads to the extinction of almost half $(\sim 0.5)$ the light that is incident on the sample at the SP resonance, whereas the samples with just the CQDs film or just the MNPs (in figure 3 ) exhibit much less extinction $(\sim 0.05$ and 0.18 , respectively) at the SP wavelength. Such pronounced extinction is the sum of light absorption in the CQDs film, absorption inside the MNPs material and light scattered outside the sample. However, extinction measurements alone do not allow a quantitative separation of the amount of energy spent in each of these effects, but they can be qualitatively analyzed with the aid of electromagnetic modeling such as the Mie theory formalism employed in this work. Figure 6 shows the two components of the extinction efficiency $Q_{\text {ext }}=$ $Q_{\mathrm{S}}+Q_{\mathrm{A}}$, the scattering $\left(Q_{\mathrm{S}}\right)$ and absorption $\left(Q_{\mathrm{A}}\right)$ efficiencies, at the SP resonance of an Au MNP, calculated with Mie theory as a function of the real part of the medium refractive index $\left(n_{\mathrm{m}}\right)$. The polarizability of the MNP at its SP resonance tends to increase [18] with $n_{\mathrm{m}}$, thus, so does its $Q_{\text {ext }}$. That is the main reason why the heights of the extinction peaks of MNPs on glass ( $n_{\mathrm{m}}=1.25$ - see figure 3 ) exhibit a three-fold 


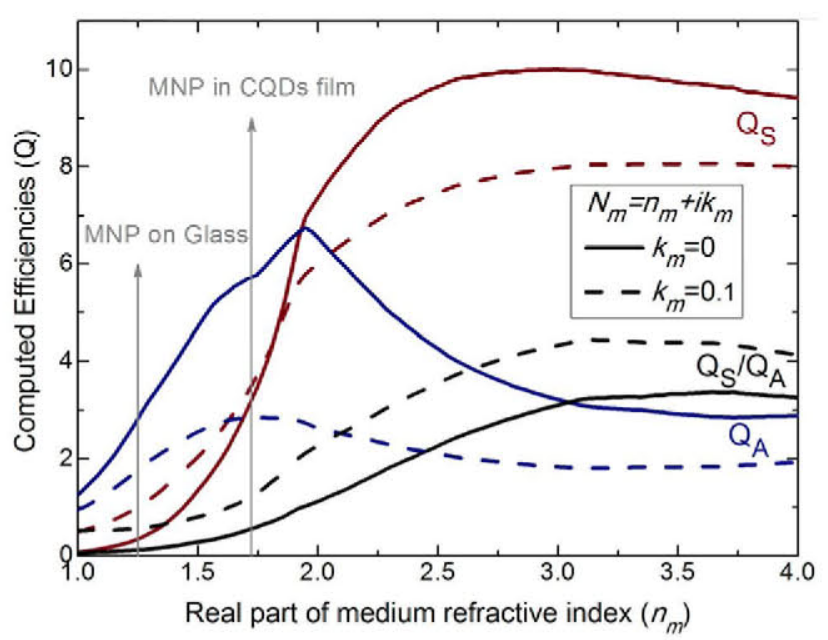

Figure 6. Scattering $\left(Q_{\mathrm{S}}\right)$ and absorption $\left(Q_{\mathrm{A}}\right)$ efficiencies at the $\mathrm{SP}$ resonance of a $50 \mathrm{~nm}$ diameter $\mathrm{Au} \mathrm{MNP}$ immersed in a uniform medium, as a function of $n_{\mathrm{m}}$. The magnitude of the polarizability of the MNP increases with increasing $n_{\mathrm{m}}$, such as the ratio $Q_{\mathrm{S}} / Q_{\mathrm{A}}$, up to $n_{\mathrm{m}} \approx 3.5$. Therefore, in general a higher $n_{\mathrm{m}}$ allows a higher absorption enhancement caused by the MNPs in their surrounding material. The dashed curves $\left(k_{\mathrm{m}}=0.1\right)$ were computed with a generalization of Mie theory for absorbing media with complex refractive indices [27], which calculates the absorption and scattering efficiencies by integrating the flow of energy at the particle surface.

enhancement after covering the MNPs with the CQDs film $\left(n_{\mathrm{m}}=1.72\right.$ - see figure 5). It can be seen from figure 6 that in the samples of figure 3 practically all the light extinction should be caused by absorption inside the MNPs, since $Q_{\mathrm{A}} \gg$ $Q_{\mathrm{S}}$ at $N_{\mathrm{m}}=1.25$. Nevertheless, when the MNPs are covered by the CQDs film there is a much higher portion of light that is scattered relative to that which is absorbed in the MNPs $\left(Q_{\mathrm{S}} / Q_{\mathrm{A}}=0.57\right.$ for $k_{\mathrm{m}}=0$ and $Q_{\mathrm{S}} / Q_{\mathrm{A}}=1.25$ for $\left.k_{\mathrm{m}}=0.1\right)$. If the medium is absorbing $\left(k_{\mathrm{m}}>0\right)$, the polarizability of the MNPs is reduced and, therefore, so also are the values of the $Q_{\mathrm{S}}$ and $Q_{\mathrm{A}}$ (as shown in the dashed lines of figure 6), but the ratio $Q_{\mathrm{S}} / Q_{\mathrm{A}}$ is higher.

In view of the above, the measured extinction peaks of figure 5 have significant contributions from both light absorption inside the MNPs and scattering to their host medium (contrary to figure 3 where extinction is dominated by absorption in the MNPs). Since the host medium absorbs the light at the wavelengths of the MNPs SP resonance, as shown in the black line of figure 5 , there is necessarily an increase in the CQDs light absorption due to the field scattered by the MNPs. Therefore, the previously mentioned enhancement in the SP extinction peak of the MNPs + CQDs samples, relative to the corresponding extinction of just the CQDs film and just the MNPs on glass, indicates that the CQDs film embedded with MNPs is absorbing a considerably higher amount of light, coming from the MNPs, than in the case where no MNPs were present in the film.

\subsection{Absorption from MNP near-field in amorphous silicon medium}

A particular advantage of the patterning procedure described in section 2 is that it enables the incorporation of the hybrid MNPs + CQDs arrays in inexpensive amorphous host materials, allowing the fabrication of nanostructured semiconductor films which are promising absorbing materials for novel solar cell designs [42] such as CQD-IBSCs. Hydrogenated amorphous or nanocrystalline silicon (a-Si:H) is an interesting candidate for the host material of the particles [43], for the following reasons:

(1) It can be deposited by low-temperature $\left(150-300^{\circ} \mathrm{C}\right)$ techniques that are gentle enough to preserve the integrity of the nanoparticles. It has been verified [3] that, by directly depositing a-Si:H on top of a CQD layer, a good electronic coupling can be achieved at the CQDs/a-Si:H interface. A low processing temperature also allows the deposition of a-Si-based devices on a wide range of low-cost substrates $[44,45]$ which can be rigid (e.g. glass), flexible (e.g. plastic) or roll-away types (e.g. polymer foil).

(2) The bandgap of the material can be controlled by doping it with carbon (a-Si $\left.i_{1-x} \mathrm{C}_{x}: \mathrm{H}\right)$ [46]. Increasing concentration of carbon $(x)$ in the alloy widens the bandgap, allowing it to be tuned from a minimum value of about $1.75 \mathrm{eV}$ $(x=0)$ to $3 \mathrm{eV}(x=1)$. Therefore, the bandgap can be matched, for instance, with the optimal value for the host bandgap of IBSCs $\left(E_{\mathrm{CV}}=1.95 \mathrm{eV}\right)$ with an appropriate carbon concentration.

(3) It is the most widely used material in thin-film solar cells. Besides enabling the deposition over large areas with reduced fabrication costs, a-Si:H provides the advantage of increased light absorptivity, which allows the reduction of the solar cell thickness making the devices less expensive and lighter (especially important for space applications).

As such, the authors consider that amorphous silicon is a promising host material for inorganic devices composed of the arrays of colloidal particles studied here. Extinction measurements were performed using a-Si thin-films (see section 6.2) as the host medium for the MNP arrays patterned using the method described in section 2 . Figure 7 shows the SP extinction peaks of MNP arrays patterned onto $30 \mathrm{~nm}$ a-Si layers supported on glass substrates, before and after being covered with an additional $30 \mathrm{~nm}$ a-Si cap layer. After depositing the cap layer, the peaks red-shift because the effective $n_{\mathrm{m}}$ surrounding the particles increases $[18,23]$. The $n_{\mathrm{m}}$ of a-Si at these wavelengths is around 4.0; however, since the a-Si layers have thicknesses comparable to the sizes of the MNPs, the measured wavelengths of the SP peaks are just slightly higher than those obtained in figure 5. This means that the real part of the effective medium surrounding the MNPs should be close $\left(n_{\mathrm{m}} \approx 1.72\right)$ in both cases.

As shown in figure $6, Q_{\mathrm{S}}$ tends to increase relative to $Q_{\mathrm{A}}$ as the refractive index of the medium increases. This implies that the ratio of the light intensity scattered by an MNP over that absorbed in its interior is higher when the MNP is embedded in a dielectric material as to when it is on a substrate in air. So, the three-fold enhancement observed in the peaks height of figure 7 indicates that there should be a 


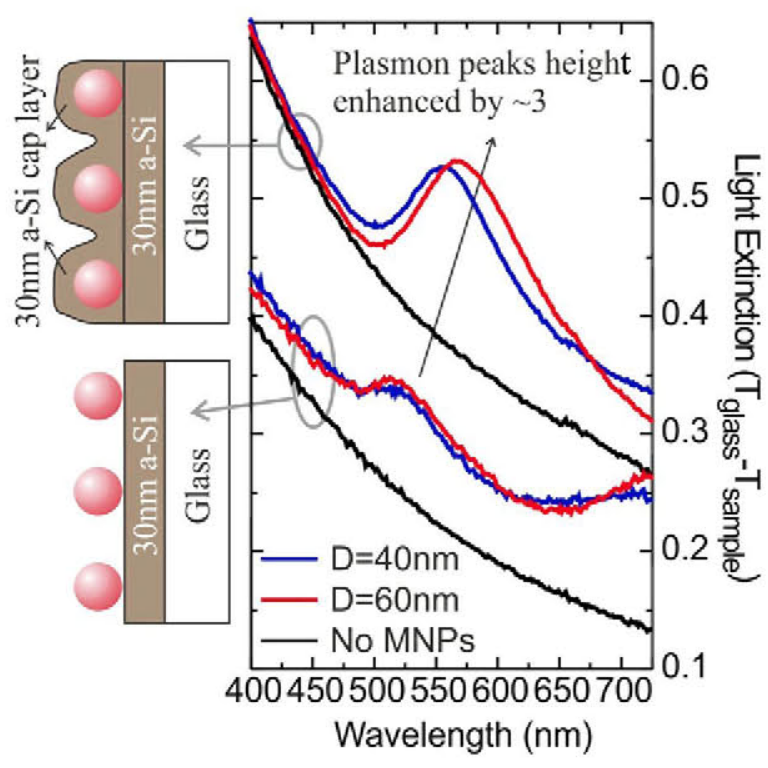

Figure 7. Measured extinction spectra of Au MNPs with distinct diameter deposited on a $30 \mathrm{~nm}$ a-Si layer over a glass substrate, before and after being covered by a $30 \mathrm{~nm}$ a-Si cap layer on top. For comparison, the extinction of the a-Si layers in both cases is also shown (black lines). The SP extinction peaks after capping the MNPs ( $\lambda=567 \mathrm{~nm}$ for $D=40 \mathrm{~nm}$ and $\lambda=579 \mathrm{~nm}$ for $D=60 \mathrm{~nm}$ ) are red-shifted relative to the peaks before capping $(\lambda \approx 530 \mathrm{~nm})$. The heights of the SP peaks, corresponding to the vertical distance between the peaks' maxima and their baselines, are increased by a factor of about 3 after covering the MNPs with the a-Si cap layer.

significant increase in the amount of light absorbed in the a-Si medium due to the MNPs scattered field, such as described in section 3.2 for the case of MNPs in the CQD film.

At the wavelengths of the SP resonances observed in figure 7 , the refractive index of a-Si has a high imaginary part $\left(k_{\mathrm{m}} \approx 0.65\right)$ which causes a pronounced absorption of the light scattered by the MNPs. Given the high absorption coefficient of a-Si at these wavelengths $\left(\alpha \approx 1.4 \times 10^{5} \mathrm{~cm}^{-1}\right)$, the propagation length (defined as length where the light intensity decays by a factor of $1 / e$ ) of the light scattered by the MNPs is only about $70 \mathrm{~nm}$. This length is close to the MNP size (D) and to the thickness of the a-Si thin-film embedding the particles. It is also considerably lower than the mean distance (3D) between the MNPs. Therefore, far-field effects, such as long-range inter-particle interactions or propagating waveguided modes, should not have a significant contribution to the observed extinction peaks. The excitation of waveguided modes in this structure would only be possible at longer wavelengths $(\lambda>0.7 \mu \mathrm{m})$ where the imaginary part of the refractive index is lower $\left(k_{\mathrm{m}}<0.1\right)$ [47].

As such, the main contribution to the extinction peaks in figure 7, after the cap layer deposition, should be the light that is absorbed in the a-Si from the fields amplified locally in the MNPs' vicinity. An effect known as near-field enhancement which has been proposed for application in various types of thin-film solar cells [18, 37, 43, 48], and also to epitaxially grown QD structures [8, 20, 21]. In this work, we're interested

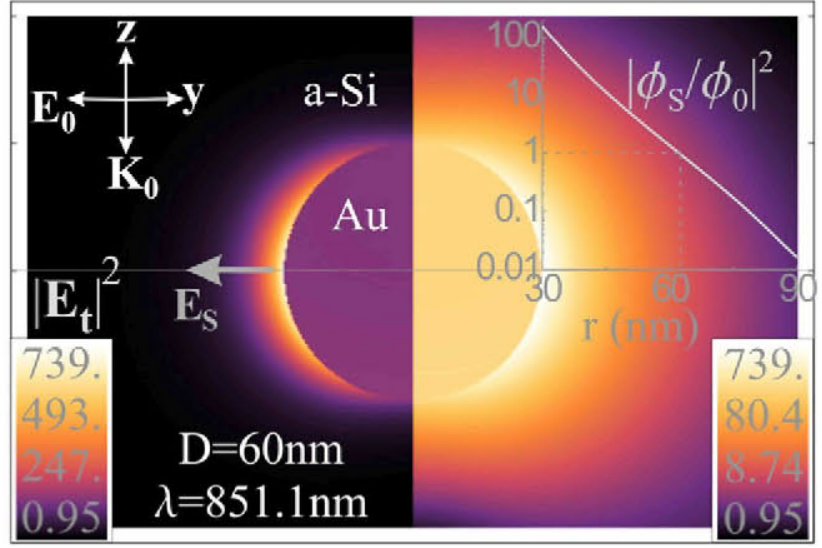

Figure 8. Distribution of the total electric-field intensity $\left(\left|\mathbf{E}_{t}\right|^{2}\right)$, in units of the incident intensity $\left(\left|\mathbf{E}_{0}\right|^{2}\right)$, inside $\left(\mathbf{E}_{\mathrm{t}}=\mathbf{E}_{\mathrm{I}}\right)$ and in the near-field outside $\left(\mathbf{E}_{t}=\mathbf{E}_{\mathrm{S}}+\mathbf{E}_{0}\right)$ a gold nanoparticle immersed in a homogeneous a-Si medium. The distribution is plotted in linear scale on the left side of the particle and in logarithm scale on the right. The origin is at the particle center. The inset plot shows the value of the electrostatic absorption enhancement $\left|\phi_{\mathrm{S}} / \phi_{0}\right|^{2}$ in the medium, along the $y$ axis outside the particle, due to the scattered longitudinal scalar photons. The wavelength of illumination $(\lambda)$ is that which allows the maximum absorption enhancement, and corresponds to the plasmon resonance of the particle in such uniform medium. The dielectric functions of Au [50] and a-Si [51] were obtained from experimental data.

in studying the near-field absorption enhancement that can be produced in the a-Si medium surrounding the MNPs, for future application in CQD-IBSCs composed of an a-Si host material. The QDs forming the IB in CQD-IBSCs shall be immersed in such intense near-field since they are patterned side-by-side with MNPs (see figure 1).

A single Au MNP embedded in an homogeneous a-Si medium produces the near-field distribution shown in figure 8 at its SP resonance, computed with Mie theory [27]. The wavelength of such resonance is higher than those measured in figure 7 because the particle is assumed to be immersed in an infinite a-Si medium $\left(n_{\mathrm{m}}=3.5-4\right)$, while in our samples the MNPs are surrounded by thin a-Si layers (effective $n_{\mathrm{m}} \approx$ 1.72 ) whose thickness is small relative to $D$ and $\lambda$.

The electric-field pattern in figure 8 is essentially dipolar, apart from a practically negligible amount of backscattering that can be observed in the slight asymmetric shape (relative to the $y$ axis) of the log-scale plot. The dipolar scattering mode is dominant because the MNP size is much smaller than $\lambda$, so it scatters in the electrostatic (non-retarded) regime [26, 49]. In this regime, the longitudinal component (parallel to the radial direction $\mathbf{r}$ ) of the scattered electric-field $\left(\mathbf{E}_{\mathrm{S}}\right)$ is dominant over the transverse component (orthogonal to $\mathbf{r}$ ) in the region of highest field intensity close to the MNP equator $[23,36](y= \pm D / 2)$. We are particularly interested in analyzing the amount of light that can be absorbed in the medium located in that region, since that is where the QDs would be preferentially located (see figures 1 and 2) [19].

The absorption of energy in the surrounding medium, from a longitudinal scattered field, cannot be calculated classically such as with far-fields which are transverse. 
A longitudinal field is a localized Coulomb field, with no associated Poynting vector, that transmits electrostatic energy to the medium by means of scalar photons created by the scattered electric potential operator $\left(\phi_{\mathrm{S}}\right)$, which is described by quantum electrodynamics [52-55]. As such, a semi-classical formalism in the Coulomb gauge [12] determines that the amount of absorption enhancement produced in a medium due to the longitudinal light scattered by an MNP, in the electrostatic regime, is given by the quantity $\left|\phi_{\mathrm{S}} / \phi_{0}\right|^{2}$, where $\phi_{0}$ is the incident potential. This quantity is plotted in the inset of figure 8 and is essentially given by the MNP polarizability squared times a factor that decays with $r^{-6}$.

In the samples of figure 7 , the majority of the a-Si cap material is deposited in the inter-spaces between the MNPs, so the enhancement in the light extinction produced by the MNPs in the a-Si should be mostly related to absorption from the MNPs' lateral near-field which is mainly longitudinally polarized. Considering that the MNPs are separated by $\mathrm{CtC}$ distances of about 3D, an order-of-magnitude estimation can be determined for this enhancement by integrating $\left|\phi_{\mathrm{S}} / \phi_{0}\right|^{2}$ over the volume outside the MNP of figure 8 delimited by a rectangular prism, centered in the MNP, with dimensions $3 \mathrm{D} \times 3 \mathrm{D} \times 2 \mathrm{D}($ in $x y z)$, and dividing by the total volume of the prism. This results in a value of 3.91 , which corresponds to an upper limit to the absorption enhancement that could be measured if the a-Si material absorbed all the longitudinal light in the MNPs' near-field. This, however, refers to the average maximum enhancement over the entire sample; but, given the evanescent profile of the scattered near-field shown in figure 8 , the absorption enhancement can be much higher at the MNP surface $\left(\left|\phi_{\mathrm{S}} / \phi_{0}\right|^{2}=160\right.$ at $\left.r=30 \mathrm{~nm}\right)$. Therefore, in hybrid arrays of CQDs and MNPs embedded in an a-Si host, the CQDs can profit from an absorption enhancement of up to two orders of magnitude.

\section{Discussion of results}

As mentioned in the beginning of section 3, light extinction corresponds to the sum of all the absorbed and scattered far-field light, so spectrophotometry measurements do not allow the determination of the gain in energy absorbed solely by the host medium due to the light scattered from its embedded MNPs. Nevertheless, as shown in section 3.2, the presence of a dielectric material surrounding the MNPs increases their polarizability and the ratio of light that they radiate through scattering relative to the light that is absorbed in their interior. As seen in figure 6, this ratio is even higher if the dielectric medium is absorbing (such as CQDs and a-Si), i.e. if its refractive index has a non-zero imaginary part $k_{\mathrm{m}}$.

In view of this, a significant portion of the extinction measured at the plasmon resonance of the MNPs embedded in CQDs (figure 5) and a-Si (figure 7) is necessarily given by the light scattered from the MNPs and then absorbed in the host material. The same, however, does not occur for the MNPs deposited on glass (figure 3 ) since, due to the low $N_{\mathrm{m}}=1.25$, the extinction should be dominated by absorption inside the metallic material of the nanoparticles.
As mentioned in section 1, for CQD-IBSCs and most opto-electronic applications, the QDs' optical properties of interest are those related to electronic transitions involving the QD ground-state. Therefore, the implementation of MNPs can only be advantageous if their SP resonance can be matched with the dots' ground-state energy.

The results presented in section 3 reveal that the plasmonic resonances of the colloidal MNPs used in this work occur at wavelengths $(\lambda=550-600 \mathrm{~nm})$ considerably lower than the wavelengths of the CQDs' ground-state $(\lambda=$ $1350-1400 \mathrm{~nm}$, shown in figure 4), both when the MNPs are embedded in films of a-Si or CQDs. So, the colloidal MNPs are designed to increase the absorption of higher energy levels in the CQDs which lie in their conduction band quasi-continuum, and not the absorption of the CQDs ground-state as would be desired. The SP resonance red-shifts when the effective $n_{\mathrm{m}}$ increases; but even in the best-case scenario of figure 8 , where the MNPs are taken to be embedded in an infinite a-Si medium, the SP wavelength $(\lambda=$ $851.1 \mathrm{~nm}$ ) is still blue-shifted from the CQDs' ground-state.

The MNPs' plasmonic resonance can be tuned to the infrared range, to wavelengths above the micrometer, by modifying their geometry and using oblate (disk-shaped) spheroidal MNPs, since the oblateness of the MNPs increases their SP wavelength.

The electric-field pattern shown in figure 8 reveals that the $D=60 \mathrm{~nm}$ MNP scatters dominantly in the dipolar electrostatic regime, since its size is much smaller than the illuminating wavelength. Therefore, the electrostatic approximation [26] can be employed to calculate the wavelength of the SP resonance of spheroidal MNPs and determine their optimal aspect ratios (width/height) that allow such resonance to be spectrally matched with the CQDs' ground-state $[12,19]$.

Figure 9 shows the spectra of the longitudinal near-field absorption enhancement $\left|\phi_{\mathrm{S}} / \phi_{0}\right|^{2}$, at the spot of highest field intensity in the MNP equator, for spherical and oblate Au MNPs in distinct media. It was verified that identical curves are obtained for the far-field $Q_{\text {ext }}$ of the MNPs. So, these curves can be compared to those measured by spectrophotometry, as presented in the inset of figure 5 which corresponds to the case of the blue curve in figure 9 (aspect ratio $=1$ ).

In order to match the SP resonance with the CQDs' ground-state, the shape of the MNPs should have an aspect ratio of 4.4 when they are embedded in an infinite a-Si host medium. The lower the real part of the refractive index of the medium the higher must be the MNPs oblateness (aspect ratio). So, for the cases of figures 5 and 7 , where the MNPs are embedded in thin-films with an effective $n_{\mathrm{m}} \approx 1.72$, the shape would have to be more elongated (aspect ratio $=19.8$ ) to allow an SP resonance at the same wavelength.

The required shape-tuning of the SP resonance is currently one of the main challenges for the application of colloidal MNPs in photovoltaics. Colloidal nanoparticles are commonly synthesized with spherical shapes and it is still difficult to controllably engineer oblate geometries in colloidal synthesis. 


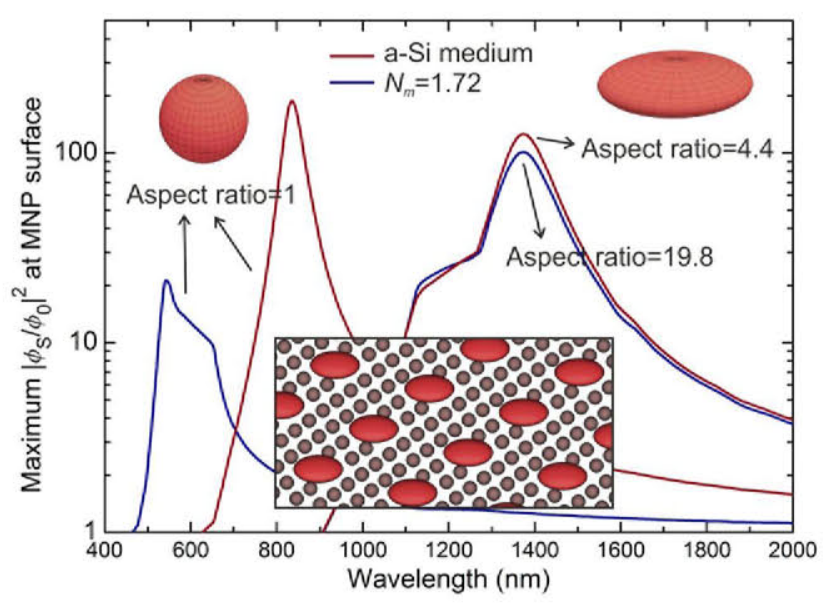

Figure 9. Absorption enhancement $\left|\phi_{\mathrm{S}} / \phi_{0}\right|^{2}$ that can be produced at the equator $(y= \pm D / 2)$ of Au MNPs with distinct shape immersed in an infinite a-Si medium or a medium with $N_{\mathrm{m}}=1.72$ (such as the thin-films of figures 5 and 7). The curves were computed with the electrostatic approximation [26] since the MNP size is taken to be much smaller than the wavelength. The aspect ratios (width/height) of the oblate MNPs are those that allow an SP resonance matched with the wavelength of the CQDs ground-state (see figure 4). The inset is a sketch of the preferential distribution of the hybrid array of oblate MNPs (red disks) and CQDs (gray spheres) in the proposed plasmon-enhanced CQD-IBSCs.

The use of more sophisticated techniques already allowed the demonstration of plasmon-enhanced QD absorption in the near-infrared range using flattened MNP shapes with elongated aspect ratios. One of the commonly used techniques is e-beam lithography (EBL) [41] which allows nanometer precision in the control of the MNPs' geometry and of the MNP-QD spacing [20]. More recently, arrays of flat MNPs were epitaxially grown on top of III-V QDs in a GaAs host material $[8,21]$. This structure exhibited large plasmon-enhanced photoluminescence from the QDs at wavelengths in the $1000-1400 \mathrm{~nm}$ range, which are close to the wavelengths of the ground-state of the PbS CQDs used here. However, these techniques are expensive, time consuming, and hardly scalable; drawbacks that together impede their use in the large-scale processing required for photovoltaic applications [23]. Therefore, the approach employed in this work is preferable since it involves low-cost fabrication methods which are compatible with mass production.

The controlled patterning of disk-shaped MNP structures on large area devices, employing inexpensive techniques, is a topic under active research nowadays [37]. In the last years there has been a considerable amount of promising advances in this area, especially due to the development of novel colloidal synthesis methods $[22,56]$ and softlithographic techniques [23] such as nano-imprint or colloidal lithography $[57,58]$.

In view of the application of the hybrid arrays in CQD-IBSCs, the MNPs and CQDs should be preferentially distributed as depicted in the inset of figure 9. Additional arrays can then be stacked on top, spaced by thin-films of host material (e.g. a-Si), to further increase the infrared light absorption in the cells. In such multilayer system, since the MNPs are bigger than the CQDs, the layers with the MNPs + CQDs array should be intercalated with one or more layers with only a CQDs array (as that of figure 2(c)), so that the lateral volume between the MNPs (the region with strongest near-field) can be filled only with CQDs embedded in the host medium.

Nevertheless, an important issue that needs to be solved, prior to the integration of MNPs and CQDs in the active region of any opto-electronic device, is the appropriate passivation of their surface since it generates defect states which can cause current degradation via carrier trapping and recombination. Therefore, both types of particles should be coated with an insulating shell material that can prevent the photo-generated carriers from reaching their surface states $[19,59]$. The use of colloids is advantageous in this respect, since their surface is accessible for chemical modification when they are in solution phase or even after being deposited. This allows, for instance, the synthesis of gold and silver MNPs coated with a silica shell $[25,35]$ having a thickness in the nanometer range. Such shell reduces the intensity of the MNPs' near-field, but can still allow them to produce absorption enhancements on the order of 10 in their surrounding medium [19]. For the case of the CQDs, core-shell $\mathrm{PbS}$ nanoparticles can be formed by controlled oxidation of the particle surface [60], creating a nanometer thin layer of oxides (of the type $\mathrm{PbSO}_{x}$ ) around the $\mathrm{PbS}$ core.

\section{Conclusions and final remarks}

Solution-based self-assembly provides a simple, scalable and low-cost method for producing ensembles of nanoparticles in a controllable manner, in order to exploit their collective properties in functional devices. A wet-coating method was developed to pattern hybrid arrays of colloidal MNPs and CQDs on the surface of various materials. The role of the MNPs is to act as optical antennas and focus the light of the appropriate wavelengths in the volume of the dots to boost their absorption.

In order to optimize the absorption enhancement that MNPs can produce in CQDs, it is important to understand how such enhancement can be influenced by the properties of the embedding material(s). The measurement of the light extinction using spectrophotometry is an efficient method to probe the plasmonic response of any light transmitting material containing MNPs. As described in section 3, such measurements allow the precise determination of the spectral position of the SP resonance and, when allied with electromagnetic modeling, they also provide information relative to the interaction between the MNPs and their surrounding medium which greatly influences the amount of light that is scattered and absorbed by the nanoparticles.

The optical results presented here show, for the first time to our knowledge, plasmon-enhanced near-field light extinction in $\mathrm{PbS}$ CQDs and a-Si thin-films patterned onto an ordered array of colloidal Au MNPs. Since such MNPs have monodispersed spherical geometries and are well-spaced in the array (i.e. there is little interaction between them), the peak 
light extinction at their SP resonance closely matches Mie theory calculations taking typical values for the surrounding refractive indices. These results are of interest for novel nanostructured photovoltaic materials composed of hybrid MNP + CQD arrays embedded in an a-Si host medium. That is one of the preferential semiconductor host materials and it can be deposited by low-temperature techniques without damaging the patterned particles array, as seen in the results of figure 7 .

In the electrostatic size regime $(D \ll \lambda)$, the propagating far-field scattered by the MNPs is generally not the major responsible for absorption enhancement in their surrounding material [18]. In this regime, the most significant enhancement in the optical fields occurs locally in the vicinity of the MNPs, through the so-called near-field enhancement. Such enhancement relies on the confinement of light at scales much smaller than the wavelength, and on the corresponding high near-field intensities caused by the excitation of surface plasmons in the MNPs [8, 20]. This effect shows great potential to increase the performance not only of QD solar cells, but also of any opto-electronic device composed of nanoscopic light absorbers [7] whose optical transitions can be spectrally matched with the MNPs' plasmonic resonances. For that, an important challenge that must be addressed in future work is the shape-control of the MNPs to fully exploit their functionality. For instance, for application in CQD-IBSCs the optimal shapes for the MNPs are oblate spheroids (disks), as shown in figure 9. Since the SP local field is quite short-ranged, especially in high refractive index media like a-Si (see figure 8), such nano-disks should be patterned in the array of the CQDs, following an arrangement as that depicted in the inset of figure 9 , which can be performed with the method mentioned in section 2 and detailed in section 6 .

\section{Experimental methods}

\subsection{Preparation of hybrid arrays of MNPs and CQDS}

The solutions of MNPs and CQDs used in the present studies were synthesized by specialized companies. The MNPs solutions were purchased from BritishBioCells and contain $\mathrm{Au}$ colloids dispersed in water and stabilized by citric acid (capping agent). The CQDs solutions were purchased from Evident Technologies and contain $\mathrm{PbS}$ colloids dispersed in 1-octanol and stabilized by oleic acid molecules.

The procedure used to deposit hybrid arrays of MNPs and CQDS, as that shown in figures 2(e) and (f), involves the following steps:

(1) The sample surface is degreased in heated $\left(\sim 60^{\circ} \mathrm{C}\right)$ baths of acetone, isopropanol and methanol ( $3 \mathrm{~min}$ in each bath).

(2) Surface is cleaned in an RCA1 solution $\left(\mathrm{NH}_{4} \mathrm{OH}(25 \%): \mathrm{H}_{2} \mathrm{O}_{2}: \mathrm{H}_{2} \mathrm{O}\right.$ in $1: 1: 5$ by volume) according to procedures described elsewhere [34]. This treatment not only removes the organic impurities but also leaves an oxidized (hydrophilic) surface which is favorable for the following functionalization step.
(3) After rinsing the sample with DI water, its surface is amino-silanized by immersing it in a $10 \%$ aqueous dilution of APTMS [(3-Aminopropyl)trimethoxysilane] for $1 \mathrm{~h}$. Then the sample is carefully rinsed with DI water and ethanol, and then baked in oven (at $\sim 110^{\circ} \mathrm{C}$ ) for $1 \mathrm{~h}$. This step functionalizes the surface with a monolayer of APTMS molecules (organic linkers) that terminate in positively-charged end groups (see figures 2(a) and (d)).

(4) A vial with the MNP solution is bath-sonicated for $10 \mathrm{~min}$ to disrupt the aggregates that are formed during the storage of the solution.

(5) The negatively-charged MNPs are deposited onto the APTMS-modified surface by dip-coating [33] the sample in the MNP solution for a few hours (or minutes if a spin-coating [32] process is used). This forms a uniformly ordered MNP array, covering the entire sample area, as that in figure $2(\mathrm{~b})$.

(6) Sample is rinsed in DI water, the degreasing step (1) is repeated and then the sample is dried with a nitrogen stream.

(7) The CQD solution is bath-sonicated for $10 \mathrm{~min}$.

(8) The CQDs are deposited by wetting the sample surface with the CQD solution for $\sim 10 \mathrm{~min}$. This forms a self-assembled CQD array covering the areas between the MNPs. The remaining CQD solution is spun out of the sample at $1000-2000 \mathrm{rpm}$ for $1 \mathrm{~min}$.

(9) Sample is rinsed in the CQDs solvent (1-octanol) and in DI water, then oven-dried to remove the excess liquid and leave only the deposited colloids.

\subsection{Deposition of CQDs and a-Si thin-films}

The thin-films of stacked CQDs were fabricated by controlled drop-drying of small volumes of the colloidal solution onto glass slides. The films constructed in this way are composed of several layers of dots, and their thickness is controlled by changing the concentration of the solution and repeating the dropping-drying cycle $[5,6,31]$. The thickness of the films was monitored by measuring it at different spots along the sample with a Talystep mechanical profilometer from Rank Taylor-Hobson.

The a-Si films were deposited with a Vaksis PVDMT/2M3T magnetron sputtering equipment. The chamber was filled with Argon (Ar) at a pressure of $7.7 \mathrm{~m}$ Torr and the RF power applied to the magnetron was $150 \mathrm{~W}$. The flux of Ar was kept constant at $30 \mathrm{sccm}$. The sample was constantly rotating during the deposition processes to ensure a uniform deposition.

\subsection{SEM and AFM characterization}

SEM images were obtained with a JEOL ISM-6335F field-emission SEM at $15 \mathrm{kV}$, in the 'Centro de Microscopía Electrónica' of 'Universidad Complutense de Madrid'. The AFM characterization was performed both with a Bruker multimode Nanoscope III and with a NT-MDT Ntegra Prima equipment. 


\subsection{Optical characterization}

The light extinction measurements were performed with a Spectro320 spectrophotometer from Instrument Systems.

\section{Acknowledgments}

This work was supported by the Spanish National Plan Project 'PROMESA' (Grant No. ENE2012-37804-C02-01). M J Mendes also acknowledges 'Universidad Politécnica de Madrid' for the scholarship 'Beca de Doctorado Homologada'.

\section{References}

[1] Tang J and Sargent E H 2011 Infrared colloidal quantum dots for photovoltaics: fundamentals and recent progress $A d v$. Mater. 23 12-29

[2] Luque A, Marti A and Stanley C 2012 Understanding intermediate-band solar cells Nature Photon. 6 146-52

[3] Sun B, Findikoglu A T, Sykora M, Werder D J and Klimov V I 2009 Hybrid photovoltaics based on semiconductor nanocrystals and amorphous silicon Nano Lett. 9 1235-41

[4] Debnath R, Bakr O and Sargent E H 2011 Solution-processed colloidal quantum dot photovoltaics: a perspective Energy Environ. Sci. 4 4870-81

[5] Arquer F P G d, Beck F J, Bernechea M and Konstantatos G 2012 Plasmonic light trapping leads to responsivity increase in colloidal quantum dot photodetectors Appl. Phys. Lett. 100043101

[6] Talgorn E, Gao Y, Aerts M, Kunneman L T, Schins J M, Savenije T J, van Huis M A, van der Zant H S J, Houtepen A J and Siebbeles L D A 2011 Unity quantum yield of photogenerated charges and band-like transport in quantum-dot solids Nature Nanotechnol. 6 733-9

[7] Konstantatos G and Sargent E H 2010 Nanostructured materials for photon detection Nature Nanotechnol. $5391-400$

[8] Nötzel R and Urbańczyk A 2012 Epitaxial self-alignment: a new route to hybrid active plasmonic nanostructures Curr. Opin. Solid State Mater. Sci. 16 59-63

[9] Luque A, Martí A, Antolín E, Linares P G, Tobías I, Ramiro I and Hernandez E 2011 New Hamiltonian for a better understanding of the quantum dot intermediate band solar cells Sol. Energy Mater. Sol. Cells 95 2095-101

[10] Luque A, Marti A, Stanley C, Lopez N, Cuadra L, Zhou D, Pearson J L and McKee A 2004 General equivalent circuit for intermediate band devices: potentials, currents and electroluminescence J. Appl. Phys. $96903-9$

[11] Mellor A, Luque A, Tobias I and Marti A 2012 The influence of quantum dot size on the sub-bandgap intraband photocurrent in intermediate band solar cells Appl. Phys. Lett. 101133909

[12] Luque A, Marti A, Mendes M J and Tobias I 2008 Light absorption in the near field around surface plasmon polaritons J. Appl. Phys. 1048

[13] Wu J, Mangham S C, Reddy V R, Manasreh M O and Weaver B D 2012 Surface plasmon enhanced intermediate band based quantum dots solar cell Sol. Energy Mater. Sol. Cells 102 44-9

[14] Mellor A, Tobías I, Martí A, Mendes M J and Luque A 2011 Upper limits to absorption enhancement in thick solar cells using diffraction gratings Prog. Photovolt. Res. Appl. $19676-87$

[15] Mendes M J, Tobías I, Martí A and Luque A 2011 Light concentration in the near-field of dielectric spheroidal particles with mesoscopic sizes Opt. Express 19 16207-22
[16] Mendes M J, Tobías I, Martí A and Luque A 2011 Near-field light focusing by wavelength-sized dielectric spheroids for photovoltaic applications Renewable Energy and the Environment Technical Digest ed O T Digest (Austin, TX: Optical Society of America) p JThC

[17] Mellor A, Tobías I, Martí A and Luque A 2011 A numerical study of Bi-periodic binary diffraction gratings for solar cell applications Sol. Energy Mater. Sol. Cells 95 3527-35

[18] García de Arquer F P, Beck F J and Konstantatos G 2011 Absorption enhancement in solution processed metal-semiconductor nanocomposites Opt. Express 19 21038-49

[19] Mendes M J, Luque A, Tobias I and Marti A 2009 Plasmonic light enhancement in the near-field of metallic nanospheroids for application in intermediate band solar cells Appl. Phys. Lett. 95071105

[20] Biteen J S, Sweatlock L A, Mertens H, Lewis N S, Polman A and Atwater H A 2007 Plasmon-enhanced photoluminescence of silicon quantum dots: simulation and experiment J. Phys. Chem. C 111 13372-7

[21] Urbanczyk A, Hamhuis G J and Notzel R 2010 Coupling of single InGaAs quantum dots to the plasmon resonance of a metal nanocrystal Appl. Phys. Lett. 97043105

[22] Nie Z, Petukhova A and Kumacheva E 2010 Properties and emerging applications of self-assembled structures made from inorganic nanoparticles Nature Nanotechnol. 5 15-25

[23] Pillai S and Green M A 2010 Plasmonics for photovoltaic applications Sol. Energy Mater. Sol. Cells 94 1481-6

[24] Haridas M, Basu J K, Gosztola D J and Wiederrecht G P 2010 Photoluminescence spectroscopy and lifetime measurements from self-assembled semiconductor-metal nanoparticle hybrid arrays Appl. Phys. Lett. 97083307

[25] Goia D V and Matijevic E 1998 Preparation of monodispersed metal particles New J. Chem. 22 1203-15

[26] Bohren C F and Huffman D R 2004 Absorption and Scattering of Light by Small Particles (Weinheim: Wiley-VCH)

[27] Fu Q and Sun W 2001 Mie theory for light scattering by a spherical particle in an absorbing medium Appl. Opt. 40 1354-61

[28] Morawiec S, Mendes M J, Mirabella S, Simone F, Priolo F and Crupi I 2013 Self-assembled silver nanoparticles for plasmon-enhanced solar cell back reflectors: correlation between structural and optical properties Nanotechnology 24265601

[29] Luque A and Marti A 1997 Increasing the efficiency of ideal solar cells by photon induced transitions at intermediate levels Phys. Rev. Lett. 78 5014-7

[30] Luque A and Martí A 2010 The intermediate band solar cell: progress toward the realization of an attractive concept $A d v$. Mater. 22 160-74

[31] Rogach A L, Eychmüller A, Hickey S G and Kershaw S V 2007 Infrared-emitting colloidal nanocrystals: synthesis, assembly, spectroscopy, and applications Small 3 536-57

[32] Leu C C, Chen S T and Liu F K 2010 Spin-coating-derived gold-nanoparticle memory J. Am. Ceram. Soc. 93 3142-7

[33] Sheu J T, Chen C C, Huang P C and Hsu M L 2005 Selective deposition of gold nanoparticles on $\mathrm{SiO}_{2} / \mathrm{Si}$ nanowire Microelectron. Eng. 78/79 294-9

[34] Mendes M J, Hernández E, Tobías Galicia I, Martí Vega A and Luque A 2010 Embedment of metal nanoparticles in GaAs and $\mathrm{Si}$ for plasmonic absorption enhancement in intermediate band solar cells 25th European Photovoltaic Solar Energy Conf. and Exhibition-5th World Conf. on Photovoltaic Energy Conversion (Valencia, Spain) pp 218-22

[35] Giersig M, Ung T, LizMarzan L M and Mulvaney P 1997 Direct observation of chemical reactions in silica-coated gold and silver nanoparticles Adv. Mater. 9570 
[36] Hägglund C and Kasemo B 2009 Nanoparticle plasmonics for 2D-photovoltaics: mechanisms, optimization, and limits Opt. Express 17 11944-57

[37] Atwater H A and Polman A 2010 Plasmonics for improved photovoltaic devices Nature Mater. 9 205-13

[38] Temple T L, Mahanama G D K, Reehal H S and Bagnall D M 2009 Influence of localized surface plasmon excitation in silver nanoparticles on the performance of silicon solar cells Sol. Energy Mater. Sol. Cells 93 1978-85

[39] Zhu S, Chen T P, Cen Z H, Goh E S M, Yu S F, Liu Y C and Liu Y 2010 Split of surface plasmon resonance of gold nanoparticles on silicon substrate: a study of dielectric functions Opt. Express 18 21926-31

[40] Mertz J 2000 Radiative absorption, fluorescence, and scattering of a classical dipole near a lossless interface: a unified description J. Opt. Soc. Am. B 17 1906-13

[41] Nishijima Y, Rosa L and Juodkazis S 2012 Surface plasmon resonances in periodic and random patterns of gold nano-disks for broadband light harvesting Opt. Express $2011466-77$

[42] Marrón D F, Martí A and Luque A 2009 Thin-film intermediate band photovoltaics: advanced concepts for chalcopyrite solar cells Phys. Status Solidi a 206 1021-5

[43] Kjeldsen M, Hansen J, Pedersen T, Gaiduk P and Larsen A 2010 Tuning the plasmon resonance of metallic tin nanocrystals in Si-based materials Appl. Phys. A 100 31-7

[44] Aguas H, Ram S K, Araujo A, Gaspar D, Vicente A, Filonovich S A, Fortunato E, Martins R and Ferreira I 2011 Silicon thin film solar cells on commercial tiles Energy Environ. Sci. 4 4620-32

[45] Filonovich S A, Alpuim P, Rebouta L, Bourée J E and Soro Y M 2008 Hydrogenated amorphous and nanocrystalline silicon solar cells deposited by HWCVD and RF-PECVD on plastic substrates at $150^{\circ} \mathrm{C}$ J. Non-Cryst. Solids $3542376-80$

[46] Conde J P et al 1999 Optoelectronic and structural properties of amorphous silicon-carbon alloys deposited by low-power electron-cyclotron resonance plasma-enhanced chemical-vapor deposition J. Appl. Phys. 85 3327-38

[47] Kroll M, Fahr S, Helgert C, Rockstuhl C, Lederer F and Pertsch T 2008 Employing dielectric diffractive structures in solar cells-a numerical study Phys. Status Solidi a 205 2777-95

[48] Lee J-Y and Peumans P 2010 The origin of enhanced optical absorption in solar cells with metal nanoparticles embedded in the active layer Opt. Express 18 10078-87

[49] Mendes M J, Tobías I, Martí A and Luque A 2010 Near-field scattering by dielectric spheroidal particles with sizes on the order of the illuminating wavelength J. Opt. Soc. Am. B 27 1221-31

[50] Palik E 1997 Handbook of Optical Constants of Solids (5 Volume Set) (San Diego, CA: Academic)

[51] Retrieved Feb. 2012 Refractive Index Database, http://refractiveindex.info

[52] Cohen-Tannoudji C, Dupont-Roc J and Grynberg G 1997 Photons and Atoms - Introduction to Quantum Electrodynamics (New York: Wiley-Interscience)

[53] Keller O 2003 Near-field optics and quantum optics: an assignation arranged by four kinds of photons J. Microsc. $209272-6$

[54] Kirkengen M, Bergli J and Galperin Y M 2007 Direct generation of charge carriers in c-Si solar cells due to embedded nanoparticles J. Appl. Phys. 102093713

[55] Keller O 2007 Near-field photon wave mechanics in the Lorenz gauge Phys. Rev. A 7611

[56] Zhang Q B, Tan Y N, Xie J P and Lee J Y 2009 Colloidal synthesis of plasmonic metallic nanoparticles Plasmonics 4 9-22

[57] Fredriksson H, Alaverdyan Y, Dmitriev A, Langhammer C, Sutherland D S, Zaech M and Kasemo B 2007 Hole-mask colloidal lithography Adv. Mater. 194297

[58] Zhang J, Li Y, Zhang X and Yang B 2010 Colloidal self-assembly meets nanofabrication: from two-dimensional colloidal crystals to nanostructure arrays $A d v$. Mater. 22 4249-69

[59] Wei G D and Forrest S R 2007 Intermediate-band solar cells employing quantum dots embedded in an energy fence barrier Nano Lett. 7 218-22

[60] Galat J, Haber J, Nowotny J and Wagner J B 1975 Studies on the interaction between oxygen and lead chalcogenide surfaces Oxid. Met. 9 497-506 